\title{
Network Teaching System and Its Appli- cations in College Mathematics Learning
}

\author{
Yalan Li \\ School of Computing Science \\ Zhongkai University of Agriculture and Engineering \\ Guangzhou, China \\ Liy12000@163.com
}

\begin{abstract}
This article describes the design concept and its application of the network teaching system of College Mathematics. By making use of the flexibility and the twoway interaction of the network multimedia, the system achieves an organic combination of the traditional teachinglearning procedures and modern multimedia network technology, which enables the students' learning become interesting and not subject to the time and space constraints, and provides students a good self-learning environment.
\end{abstract}

Keywords: network; multi-media; learning system; college Mathematics

\section{Introduction}

With the increasing popularity of multimedia technology and the Internet, information technology is changing the mode of production, work, life and learning of the human society. Multimedia and the Internet and other new information technologies have shown great potentials in the field of education. As one of the most important basic courses in the engineering colleges, the learning of College Mathematics is not just learning for knowledge, it is also a training of the students' ability and quality. With its comprehensive content, large teaching time span, high density of knowledge and abstract principle, the course of College Mathematics has less glamour to attract the students. Thus, to achieve a better teaching-learning effect, to make the rich connotation of some mathematical principles and the state-of-the-art teaching ideas of the teachers fully expressed, the traditional teaching methods have not been adequate and it is very urgent for us to make an organic combination of the traditional teaching-learning procedures and modern multimedia network technology.

Currently, there are many multimedia courseware made by the use of multimedia teaching technologies, but most of which are mainly for teaching, rather than students' self-learning. Based on the design principle of student-centeredness, the network teaching system for College Mathematics makes a full use of the new technologies such as multimedia and networking, and integrates the various teaching steps, which greatly stimulates students' learning interests, effectively supports students' personalized learning and the fostering of students' ability and quality.

\section{The Overall Structure and Design Ideas of the System}

Combined with all the traditional teaching learning aspects, the entire learning 
system is divided into five sub-systems: classroom learning, tutoring, assignments, training, online testing, and extracurricular reading.

As the most basic form of students' knowledge learning, classroom learning is an important channel of access to knowledge. The sub-system of classroom learning is designed mainly for the purpose of facilitating students' after-school review, and consisted with three parts: course content, multimedia courseware and animation demo. Through the three levels of easy-to-digest and illustrated explanations from the abstract to the image, it makes the boring mathematical knowledge become interesting, and mobilizes the enthusiasm of the students from all aspects.

As an important part of the teaching process, tutoring is the continuing, supplementing and improving of the classroom lectures. The subsystem consists of two parts: doubts-clearing-up and onlineQ \& A. The widespread problems existed in students' learning mathematics and the common errors accumulated in the years of teaching practice are incorporated into the part of doubts-clearing-up of the subsystem. In the meanwhile, online-Q \& A is set up to enable students to ask questions and discuss issues online.

Workbook is not only one of the important means to familiarize students with the learning contents, master the learning methods, and improve the learning capacity, but also the most basic form for the teacher to get acknowledgement of student learning, master teaching schedule, and check the effectiveness of teaching. The workbook subsystem is divided into three parts, namely text exercises, online tasks, and online exercises. In the part of "text exercises" fixed jobs in each lecture are set for students to master the basic concepts of this course, the basic methods and the basic computing, the students are required to submit their work on time. In the part of "online tasks", real-time operating tasks are set up online based on the previous study and the specific circumstances of the students. The process of answer extraction and the problem resolution will be posted online along with the progress of teaching in a timely manner. In the part of "online exercises", a large number of topics of all kinds are selected among the textbooks of higher mathematics from home and abroad to provide students an easy access to independent practicing and answer-checking at any time.

Exam is an important means of prompting students to study hard, and also an important basis to measure the learning effect. According to the knowledge structure of College Mathematics and the course schedule, the subsystem of online testing is designed, including unit test, mid-term test and finalterm test with answers provided for students to self-test their learning effect. The subsystem also sets up the section of test papers for teachers to post new questions online, and for students to submit papers.

The subsystem of extracurricular reading: students can learn about some of the personal stories related to mathematics and background knowledge about mathematics.

\section{The Choice of the System- developing Tools}

Presently the softwares available for the production of multimedia learning system mainly include Authoware, Flash, Powerpoint, Deamweaver and etc. Each has its own merits. In the entire network learning system of College Mathematics, different learning areas have their own characteristics. For example, in the course study some animations are needed to make a flexible and vivid demonstration, enabling the contents, which are difficult for teachers to express and ambiguous for students to understand, easily resolved; 
the section of online Q \& A has the interactive feature of students asking questions and teachers posting their answers; and the entire learning system is available online and thus easy to spread. By comparison, Deamweaver is selected as the developing platform, and PowerPoint and Flash as the complements, making the network learning system of College Mathematics one of complementary functions, friendly production interface, and flexible operation.

\section{Realization of the System Functions}

The homepage of each subsystem is of the structure of up, lower left, and lower right. The labels of the five subsystems are located at the top, to facilitate the quick jump between the subsystems. The lower left of the page, which accounts for about a quarter of the screen, is for the demonstration of each chapter, section, and knowledge structure. The lower right works as the main window. When a certain knowledge point is selected, the corresponding content is automatically displayed in the main window of the subsystem. The homepage structure is produced by Dreamweaver, while when it is time to display the content of each homepage, other different software tools are employed according to the features of different content.stem Functions.

\subsection{Implementation of Classroom Learning}

\subsubsection{Course Content}

The section of the course content involves the word processing. In order to facilitate the input of mathematical formulas, the course content is edited into word documents, then transformed into web documents.

\subsubsection{CAI Courseware}

Although CAI courseware is an important teaching aid, it is unsuitable to make it too large and all-encompassing. The teaching content, which is abstract and uneasy to be expressed clearly by the use of words, can be conveyed effectively with the help of multimedia tools. And then when matched with the corresponding graphic, animation and appropriate hyperlink to make a courseware, it will be much easier to achieve an ideal teaching effect, making the classroom lectures vivid and conducive, enabling the students have a better understanding and a deeper impression of the learning content. The production of CAI courseware is achieved mainly by PowerPoint, combined with Flash.

\subsubsection{Animated Demo}

Animations in this section are all made by Flash. They are used to demonstrate the change process of some phenomena in the content of higher mathematics; construct all kinds of space graphics to show their combination and split, mutual positional relationship of the whole and the part. For example, animation can be used to display the geometric meaning of the guide number: the secant gradually changes to the maximum position to get the tangent, and then arrive at the derivative of the function at a point which means the slope of the curve at that point in geometry. Another example is the discussion of the shape of quadric surface by the method of cut-off mark: with animation, the dynamic process of intercepting the surface of a series of planes parallel to the coordinate plane can be easily demonstrated by observing the change of the plane and the cut-off marks of the hyperboloid to understand the whole picture of the surface. Here not only some animations related to classroom content are 
provided, some operating exercises or other counseling books also employ some animated graphics for the students to get reference at any time.

\subsection{The Implementation of Tutoring}

\subsubsection{Doubts Clearing-up}

In the section of doubts clearing-up, problems and doubts in the mathematics curriculum are edited in the form of chapter. Q \& A in each chapter is on one page and displayed in the main window as required. On the top of the page all the questions are presented by using the roller and the answers are displayed at the bottom of the page. When a problem at the top is selected, the content at the bottom will be automatically updated. This functionality is achieved by employing the technology of "display" and "hide" in Dreamweaver.

\subsubsection{Online $\mathbf{Q} \& \mathrm{~A}$}

Online Q \& A is for students to post questions directly online, and receive the corresponding answers of teachers. It plays an irreplaceable role in strengthening the exchange of teachers and students and helping students to make clear where the problem is and get answers they really need. In this process database access is involved, and dynamic database access technology ASP is applied accordingly to achieve real-time, interactive, and $\mathrm{dy}$ namic function.

The core of the design principles in this section is handiness and practicality, helping students to make use of online Q $\&$ A easily and conveniently. In this section four items -- question-asking, issues list, answer-browsing and teacher management, are designed to achieve the functions of students asking questions, displaying questions and checking their status -- whether the questions are an- swered or not, watching the questions being answered by the teachers, and teachers answering questions respectively.

Unavoidably the questions related to the college mathematics curriculum contain mathematical formulas, and usually such formulas can not be input directly in the web page. Therefore, accessory items are designed respectively in the title areas of students asking questions and teachers answering questions, which enables the narrative mathematical questions or answers be directly input in the title area. In the meanwhile, the questions containing mathematical formulas can be edited into document or picture otherwise. For example, a document compiled by Word can be submitted to post questions and answers by the function of attachment upload and download. When there are attachments of questions asked by students or answered by teachers, the accessories will also appear in the corresponding browsing areas for students to download and read. To have a better understanding about the students and enhance contact with them, the students are required to provide their register name, class, problem title and other information alike. The tile area of questions is to describe the issues raised by students, and to make the question searching convenient, part of the contents in the tile area will be displayed in the list of questions. Teachers view the list of questions; learn the status of the questions - what questions are asked and whether or not the questions are answered; then get access to the platform from the portal of teacher management and respond to the students' questions. And by viewing the question list and the question status, students are able to browse the answered questions. 


\subsection{The Implementation of Workbook}

\subsubsection{Text Exercises, Online Tasks}

The part of "workbook" deals with the fixed jobs in each chapter, and thus seems quite similar to the part of "classroom learning" in the processing mode. "Online tasks" contains the newly added exercises according to the instant teaching and learning conditions, and is equipped with three annexes items: assignment-setting, job-submitting, and answer-downloading. The system still employs the technology of dynamic database access ASP to realize the dynamic and interactive functions of teacher assigning and student submitting.

\subsubsection{Online Exercises}

"Online exercises" is for students to practice on their own after school, so when the designing of its interface is concerned, it is not only necessary to take into account the facility for students to finish their tasks, the easy access to the answer-checking should also be seriously considered. The types of questions in the exercises include blank-filling, multiplechoice, $\mathrm{T}$ or $\mathrm{F}$, and question answering. In blank-filling students can type the answers in the blank areas, which is realized by the use of text field.

There is an answer button for each question. Students can check the correctness of the question by clicking on the answer button. Multiple-choice usually takes its normal form: once the student clicks on A, B, C or D to make a selection, a onMouseDown event is triggered, the browser will check the trigger and call the appropriate JavaScript code to lead to the occurrence of an action - the appearance of a corresponding icon of judgment. If the choice is correct, then the finger chart "V" appears, indicating the correct- ness of the choice, and if the choice is not correct, there is other iron available to demonstrate the wrongness of the choice. When the mouse leaves the clicking area, the question surface restores to its original look. Question-answering is different from the previous two, in that it needs to examine students' train of thought in answering questions. Therefore, the problem-solving ideas and the process are all provided in this part: when student clicks on the answer button, the answer is displayed in a new window of smaller size, helping student to view the answer with the original question aside.

\subsection{The Implementation of Online Test}

Different from doing exercises, there is no need to check the answer for each completed question in the section of "online test". When the whole paper is completed, student can click on the answer button and get the original questions and the process of their resolution. The question and the answer are all displayed in the main window of online test, and their revealing \&concealing can be controlled. The part of test papers sets up the items of paper-releasing, paperdownloading, and paper-submitting, for teachers or students to issue new papers, download and submit papers.

\section{Conclusion}

By using the technology of the network multimedia, the flexible and easy-to-learn system is produced in line with the principle of student learning. It is not only an e-learning system for students to conduct after-school study, but also a teaching system for teachers to assist classroom teaching. It applies to the entire teaching process and gets updated and expanded along with the changing and updating of the learning content and knowledge areas, 
providing a broad space for learning and a rich learning environment for students.

\section{References}

[1] Department of Mathematics of Tongji University, "Higher Mathematics, 4th ed", Beijing: Higher Education Press, pp.77-83, 1996.

[2] Kekang He, "E-learning and the Deepening of the College Teaching Reform", China Audio-Visual Education, pp.46-50, 2002.

[3] Lianghai Wu, "Design and Development of Web-based Multimedia Courseware", Era of Computer, pp.49-53, 2011.
[4] Li Han, "Analysis of the Teaching Model Based on Moodle Platform", Primary and Secondary Audio-visual Education, pp.23-27,2007.

[5] Meiyu Lu, "Information-based Curriculum Design and Application Based on Platform", Journal of Guangxi Normal University, pp.13-16, 2008.

[6] Quanwei Wu, Xuyi Wang, "The Applications Tutorial of Dreamweaver MX 2004", Beijing: China Railway Press, pp.234-256, 2004.

[7] Xingming Zhou, Daowei Tu, and $\mathrm{Xi}$ feng Lin, "Design and Production Instances of CAI Courseware", Wuhan: Huazhong University of Science and Technology Press, pp.40-42, 2010. 